\title{
EL ESTILO EXPLICATIVO PESIMISTA Y LA AUTOEFICACIA OCUPACIONAL: UN ANÁLISIS CUANTITATIVO BICULTURAL
}

Pessimist explanatory style and occupational self-efficacy: A bicultural quantitative analysis

Daniel Arturo Cernas Ortiz ${ }^{1}$

Fecha de recepción: 16 de febrero del 2018

Fecha de aceptación: 28 de febrero del 2018 


\section{Resumen}

El objetivo de este trabajo es examinar la relación entre el estilo explicativo pesimista y la autoeficacia ocupacional. También, determinar si la fuerza de este efecto varía entre México y Estados Unidos. Se aplicó un cuestionario autoadministrado en ambos países ( $n$ en México = 282, EE.UU. = 275). Las hipótesis se probaron utilizando regresión lineal jerárquica y análisis de moderación. Los resultados indican que el estilo explicativo pesimista tiene una relación negativa con la autoeficacia ocupacional y que esta relación es más pronunciada en Estados Unidos que en su vecino del sur. En conjunto, los hallazgos sugieren que las atribuciones malsanas de eventos negativos tienen el poder de inhibir las experiencias directas de éxito y, por lo tanto, el desarrollo de las creencias de autoeficacia en el dominio ocupacional. Además, la cultura nacional puede amplificar tal efecto haciéndolo más negativo en Estados Unidos que en México.

Palabras clave: Estilo explicativo, autoeficacia, atribuciones, cultura nacional

\section{Abstract}

The aim of this research study is to examine the relationship between pessimist explanatory style and occupational self-efficacy. Also, to assess whether the strength of this relationship varies between Mexico and the United States. A self-administered survey was applied in both countries (Mexico's $n=$ 282, U.S.A. 's $n=275$ ). Hierarchical linear regression and moderation analysis were employed to test the study hypotheses. The results indicate that pessimist explanatory style has a negative relationship with occupational self-efficacy and that this relationship is more pronounced in the United States than in its southwestern neighbor. On the whole, the findings suggest that unhealthy attributions for negative events have the power to inhibit enactive mastery and, therefore, the development of self-efficacy beliefs in the occupational domain. In addition, national culture could amplify such effect making it more negative in the United States than in Mexico.

Keywords: Explanatory style, self-efficacy, attributions, national culture 


\section{Introducción}

\section{El estilo explicativo pesimista}

$\mathrm{E}$

n el trabajo y las organizaciones, las atribuciones han sido vinculadas a fenómenos impor-

tantes como la motivación y la satisfacción. Spector (1982), por ejemplo, indica que el locus

de control interno incrementa la iniciativa y la motivación para el éxito. Sin embargo, el locus de control captura sólo parcialmente las atribuciones personales debido a que ignora la estabilidad y la globalidad de las causas. Así, mientras que una persona puede atribuir un determinado fracaso a sus habilidades innatas, otra podría atribuirlo a su esfuerzo. Como está documentado (Bridges, 2001; Weiner, 2010), aunque ambas son causas internas, la persona que atribuye el fracaso a una causa inestable (el esfuerzo) estará más motivada a mejorar que la que lo hace a una estable (la habilidad).

No obstante, y de acuerdo con el modelo reformulado de la indefensión aprendida (Abramson, Seligman y Teasdale, 1978), el estilo explicativo es un esquema de atribuciones de más relevancia para entender padecimientos psicológicos perniciosos como la depresión. Las personas tenemos estilos personales diferentes para explicar lo positivo y lo negativo que nos pasa (Peterson y Seligman, 1984). Algunos individuos desarrollan la tendencia a atribuir sus eventos negativos a causas internas (p.ej. pasa por mi culpa), estables (p.ej. me pasará siempre) y globales (p.ej. pasa en todos los ámbitos de mi vida) implicando que tales eventos abundarán en el futuro, que son inevitables y que es imposible hacer algo al respecto. Estos mismos individuos también enfatizan causas externas, inestables y específicas para eventos positivos. En conjunto, esta forma de pensar, llamada estilo explicativo pesimista (Abramson $e t$ al., 1978), puede ayudar a entender fenómenos organizacionales de manera más completa debido a que incluye, además de la internalidad, la estabilidad y la globalidad de las causas (Furnham, Sadka y Brewin, 1992). Proudfoot, Corr, Guest et al. (2009) sugieren que el estilo atribucional tiene efectos importantes en la satisfacción laboral, la autoestima, el bienestar subjetivo, la productividad y la rotación de personal. Miralles y Navarro (2016) argumentan que el estilo explicativo podría interactuar con la evaluación cognitiva de eventos ocurridos en el trabajo para influenciar el afecto experimentado en la organización. Por supuesto que estos hallazgos hacen eco de los reportados por Seligman (1990) con respecto a que el estilo explicativo pesimista estaría relacionado con un desempeño laboral menor al que se obtendría con un estilo explicativo más optimista.

\section{La autoeficacia ocupacional y el estilo explicativo pesimista}

La autoeficacia es un concepto central para entender la motivación y el éxito. Este término se refiere a la estimación personal de la capacidad para lograr un determinado nivel de desempeño en una tarea específica (Gits y Mitchell, 1992). Con base en los postulados de la teoría social-cognitiva, aquellas personas cuyas 
creencias de autoeficacia son sólidas, son más propensas a ponerse metas retadoras y a lograrlas en forma efectiva (Bandura y Locke, 2003). Por supuesto que entre más retadoras sean las metas, más alto es el desempeño en una determinada tarea (Locke y Latham, 2009). De tal suerte, en el trabajo y las organizaciones, la autoeficacia es un término importante porque está relacionado significativamente con la motivación, el establecimiento de metas y el desempeño (Judge y Bono, 2001; Park y Jung, 2015).

Para entender mejor las implicaciones de la autoeficacia y realizar comparaciones más efectivas entre diferentes tipos de trabajo, Schyns y von Collani (2002) postulan el término de autoeficacia ocupacional. Estos autores definen su concepto como la creencia en que uno puede movilizar los recursos necesarios para atender satisfactoriamente las exigencias del dominio ocupacional. La autoeficacia ocupacional está relacionada positivamente con la satisfacción laboral y el compromiso organizacional (MerinoTejedor, Fernández-Ríos y Bargsted-Aravena, 2015; Rigotti, Schyns y Mohr, 2008), además de explicar varianza adicional en la satisfacción laboral más allá de la explicada por las creencias de autoeficacia generalizada (Schyns y von Collani, 2002).

Bandura (1977) indica que las experiencias directas de éxito (enactive mastery) son la principal fuente de autoeficacia. En otras palabras, tanto en tareas específicas como en ocupaciones, en lo principal la gente llegamos a creer que somos capaces de lograr un cierto desempeño cuando lo intentamos y lo logramos. El éxito repetido hace que se genere confianza en la eficacia personal y que germine la creencia de que uno es capaz de ejercer una ocupación en forma exitosa (Bandura y Locke, 2003). Sin embargo, es innegable que el fracaso es inherente a toda actividad humana y que de él se aprehenden lecciones que, eventualmente, aumentan la posibilidad de resultados positivos. En este punto es importante el estilo explicativo pesimista pues cuando la gente no tiene éxito y atribuye tal situación a causas internas y estables (p.ej. no tengo habilidad), además de globales, deja de intentar lograr lo que se propone, no procura aplicar el aprendizaje para evitar los errores y, en consecuencia, se aleja del éxito (Gits y Mitchell, 1992). Obviamente, este patrón cognitivo y conductual es más probable que aumente las creencias de incompetencia que de las de autoeficacia en la realización de una ocupación. Weiner (2010) sugiere que la motivación para remontar un fracaso se mantiene, e incluso puede intensificarse, cuando la gente atribuye su experiencia a causas internas pero inestables (p.ej. no hice el esfuerzo suficiente).

\section{La importancia de la cultura nacional}

En términos simples, la cultura nacional se entiende como los valores, las normas y las suposiciones compartidas por un grupo de personas en un espacio y un tiempo determinado. La cultura nacional es importante porque interactúa con los rasgos individuales para delinear el comportamiento (McCrae, 2000). Por implicación, la relación entre diversos constructos psicológicos y laborales podría variar en fuerza entre diferentes culturas. Como ejemplo, Cernas-Ortiz y Davis (2016) encontraron que la relación entre la perspectiva de tiempo y la satisfacción laboral es más débil en Estados Unidos que en México. También, Urien, Osca y García-Salmones (2017), muestran que la ambigüedad del rol se relaciona menos negativamente con la satisfacción laboral en España que en México.

Para Hofstede, Hofstede y Minkov (2010) la cultura de los países difiere en seis dimensiones: masculinidad (la competitividad y la asertividad son prevalentes en la sociedad), evasión de la incertidumbre 
(la gente prefiere lo conocido, las reglas y la estructura), distancia de poder (las diferencias sociales en estatus y privilegios se esperan y se aceptan), colectivismo (la gente se concibe a sí misma como parte de un grupo social y las metas de éste tienen primacía sobre las individuales), orientación al corto plazo (la gente pone énfasis en el aquí y ahora) e indulgencia (tendencia social a la gratificación y al hedonismo). Estas dimensiones caracterizan a las culturas y sirven para hacer predicciones sobre las posibles variaciones en la relación entre constructos psicológicos en diversas sociedades (Hofstede, 2015).

México y Estados Unidos son dos países en los que la relación entre constructos psicológicos podría variar en fuerza. Después de todo, Hosftede et al. (2010) reportan que la cultura mexicana y la estadounidense difieren en todas sus dimensiones siendo México consistentemente más alto que Estados Unidos. Estos hallazgos no son aislados pues el Proyecto GLOBE (House, Hanges, Javidan et al., 2004) arrojó resultados similares. Como explicación de las diferencias, Octavio Paz (2009) argumenta que México y su vecino del norte son herederos de versiones diferentes de la cultura occidental. Aunque sus diferencias culturales han sido estereotipadas y ampliamente exageradas en el cine y los medios masivos de comunicación (Cortés, 1989), es innegable que mientras que la cultura mexicana está fuertemente influenciada por la cultura indígena, española y católica, la estadounidense tiene fuertes raíces en los valores ingleses y protestantes (Curzio, 2013).

Con base en lo anterior, la fuerza de la relación entre el estilo explicativo pesimista y la autoeficacia ocupacional podría variar entre México y su vecino del norte. Aunque varias de las dimensiones de Hofstede et al. (2010) formarían una constelación cultural que ayudara a explicar la diferencia entre los países, dos dimensiones: indulgencia y orientación al corto plazo pueden ayudar a entender el fenómeno más parsimoniosamente. En conjunto, ambas dimensiones se relacionan con la tendencia social a la gratificación de los placeres en el aquí y el ahora (Hofstede et al., 2010). De tal suerte, no resulta tan sorprendente que México se reporta como un país de alto bienestar subjetivo (felicidad) (OECD, 2017), incluso mayor que el de Estados Unidos cuando se controla por el nivel de ingreso económico per cápita (Dienner y Diener, 1995; Minkov, 2009). Por ello, y con base en cierta evidencia que muestra al estilo explicativo como un predictor de la felicidad (Cheng y Furnham, 2001), resulta lógico que la tendencia de la cultura mexicana a la felicidad estaría relacionada con el optimismo a nivel social. Ya que en una sociedad optimista la atribución de los eventos negativos a causas internas, estables y globales es menos potente (Oettingen, 1995a), la relación entre el estilo explicativo pesimista y la autoeficacia ocupacional sería menos fuerte en México que en Estados Unidos.

Reforzando lo anterior, Oettingen y Morawska (1990) argumentan que la tendencia al pesimismo es visible en ciertas manifestaciones materiales de la cultura, por ejemplo, en el muro de Berlín. Ellos indican que una sociedad que espera más cosas buenas que malas en su provenir no tendría mucha razón para forzar a las personas a permanecer en su país de origen. Resulta interesante el paralelismo de esta situación con la intención del actual gobierno estadounidense de construir un muro en su frontera sur. Tal pareciera que (debido a los mexicanos) muchas cosas malas podrían pasar en ese país y, por ello, los extranjeros deben permanecer alejados.

\section{Propósitos de este estudio}

Con base en lo anterior, el objetivo de este trabajo es probar la relación negativa entre el estilo explicativo pesimista y la autoeficacia ocupacional. Además, explorar si esta relación varía entre México y Estados Unidos siendo más fuerte en el segundo país. Formalmente, se postula lo siguiente: 
Hipótesis 1. El estilo explicativo pesimista está relacionado negativamente con la autoeficacia ocupacional.

Hipótesis 2. El estilo explicativo pesimista está relacionado más negativamente con la autoeficacia ocupacional en Estados Unidos que en México.

\section{Método}

\subsection{Participantes}

Los datos se recolectaron en una universidad pública del centro de México y una del suroeste de Estados Unidos. 299 estudiantes de Maestría en Administración (MBA) participaron en México y 290 en Estados Unidos. Las muestras no fueron aleatorias. Después de eliminar las respuestas incompletas, la base de datos final en México se compuso de 282 personas (Hombres $=126$, Mujeres $=156$ ) y la de Estados Unidos de 275 (Hombres $=141$, Mujeres $=134)$. La mayoría de los participantes tenía un empleo de tiempo completo al momento del estudio (México $=72.4 \%$, EE.UU. $=65 \%$ ). Pocas personas reportaron estar desempleadas (México $=3.8 \%$, EE.UU. $=12.5 \%$ ), aunque todas contaban con experiencia laboral previa. Ninguna persona indicó ser (o haber sido) obrero o ejecutante de tareas manuales. La edad promedio fue de 31.32 años en México y de 29.34 en EE.UU.

\subsection{Procedimiento}

Se empleó un cuestionario impreso en papel que se entregó a los estudiantes durante sus horas de clase. No se solicitaron datos personales como nombre o número de identificación para infundir confianza en la encuesta y controlar sesgos de respuesta como la deseabilidad social o las correlaciones ilusorias. Los desempleados contestaron tomando en cuenta su experiencia laboral más reciente. El diseño de investigación fue cuantitativo, no experimental y correlacional.

Las escalas de medición fueron traducidas de su original en inglés al español (versión mexicana) antes de la aplicación del cuestionario en México. Todos los reactivos (ítems) se tradujeron a significados comparables y en ningún caso fueron traducciones literales. Uno de los autores de este trabajo tradujo los reactivos al español. Después, los reactivos fueron retraducidos al inglés por un individuo ajeno a la investigación. Se discutieron las discrepancias entre la versión original en inglés y la retraducida del español para corregir las traducciones dudosas. Este proceso se siguió hasta que las versiones originales y las retraducidas se consideraron iguales (sólo dos iteraciones).

\subsection{Instrumentos}

Estilo explicativo pesimista. La escala de cuatro reactivos para medir atribuciones de eventos negativos relacionados al trabajo fue modelada de acuerdo al cuestionario de estilo atribucional ocupacional de Furnham et al. (1992). Se presentó a los respondientes cuatro eventos hipotéticos (p.ej. "Recientemente te enteraste de que no recibirás una promoción que has estado esperando por mucho tiempo"). Desde su propia perspectiva, cada respondiente escribió la "causa principal" del evento. Posteriormente, utilizando 
una escala tipo Likert de siete puntos, cada persona evaluó la causa del evento en tres dimensiones: interno - externo ( 1 = "Totalmente debido a otras personas o circunstancias, a 7 = "Totalmente debido a mí), estable - inestable ( 1 = "Nunca estará presente otra vez", a 7 = "Siempre estará presente") y específico - global ( 1 = "Influencia sólo esta situación en particular", a 7 = "Influencia todas las situaciones de mi vida"). Se obtuvo un puntaje por evento sumando los tres reactivos atribucionales de cada uno. Un puntaje global de estilo explicativo por persona se obtuvo promediando los puntajes totales de cada individuo en los cuatro eventos. Entre más alto es el puntaje global, más fuertes las atribuciones negativas de eventos negativos de la persona. Para este trabajo, la confiabilidad ( $\alpha$ ) de la escala en cuestión fue de 0.70 en los dos países considerados.

Autoeficacia ocupacional. Para medir esta variable se utilizó la forma corta de la escala de autoeficacia ocupacional de Schyns y von Collani (2002). Esta escala tiene ocho reactivos en un formato Likert anclado en 1 (para nada cierto) y 7 (muy cierto). Un ejemplo de esta escala es la oración "Logro las metas que me propongo a mí mismo(a) en el trabajo". La confiabilidad ( $\alpha$ ) de la escala fue de 0.89 en México y de 0.91 en EE.UU. Rigotti et al. (2008) validaron el uso de estas preguntas en contextos hispano (España) y angloparlantes (Reino Unido).

Variables de control. En las pruebas de hipótesis se controló por la edad, el sexo (Hombre $=1$, Mujer $=2$ ) y la situación laboral (Empleado $=0$, Desempleado $=1$ ) de los participantes. Estas variables fueron consideradas debido a que podrían afectar directamente los niveles de autoeficacia ocupacional y, por lo tanto, representarían explicaciones alternativas a la covariación entre el estilo explicativo pesimista y la autoeficacia ocupacional.

\subsection{Análisis}

Validez. Se utilizó un análisis factorial confirmatorio (AFC) en LISREL (8.8) que incluyó la comparación de dos modelos por cada una de las muestras utilizadas. En el primer modelo se forzó a todos los reactivos a cargar en un solo factor que reprodujera la matriz original de covarianza. Luego, en el segundo modelo, los reactivos fueron asignados al constructo teórico al que pertenecen (estilo explicativo pesimista o autoeficacia ocupacional). Por cada muestra se comparó ambos modelos para determinar si la solución que debería reflejar mejor la estructura original de covarianza (la de dos factores) era significativamente superior a una alternativa donde no hubiera diferenciación entre constructos (la de un factor). La prueba de diferencias en $\chi^{2}$ se utilizó para determinar si la solución de dos factores difería significativamente de la de uno.

Adicionalmente, se realizaron pruebas de invarianza de medición para determinar si los constructos involucrados eran equivalentes en términos configurales y métricos entre las dos muestras. Estas pruebas se realizaron siguiendo las recomendaciones de Byrne (2006).

Descriptivos. Se calculó la media y la desviación estándar de las variables. También se calcularon correlaciones bivariadas como paso preliminar a la prueba de las hipótesis.

La varianza del método común. Se utilizó el método de Lindell y Whitney (2001) para determinar la presencia de este fenómeno que podría alterar artificialmente las relaciones estadísticas encontradas.

Prueba de hipótesis. Para probar la Hipótesis 1 se utilizó regresión jerárquica múltiple por el método 
de mínimos cuadrados ordinarios. La prueba de la Hipótesis 2 se realizó en dos fases. En primer lugar se analizó el efecto moderador del país en un modelo de regresión jerárquica múltiple que incluyó el efecto principal del estilo explicativo pesimista, una dummy por país (México $=0$, EE.UU. $=1$ ) y la interacción entre el estilo explicativo pesimista y la dummy por país. Esta interacción se formó por el producto simple de las variables. Antes de obtener este producto, el estilo explicativo pesimista se centró a la media para evitar problemas de multicolinealidad con su efecto principal. Después se realizó la prueba de Chow para determinar con mayor precisión si los coeficientes de regresión entre las dos muestras se podían considerar significativamente diferentes.

\section{Resultados}

\subsection{V alidez}

El análisis factorial confirmatorio indica que, en ambas muestras, la solución de dos factores es superior a la de uno (México $\Delta \chi^{2}=603.78$, EE.UU. $\Delta \chi 2=274.98$ ) siendo esta superioridad significativa a nivel de 0.01. En ambos casos, la solución de dos factores muestra indicadores de bondad de ajuste adecuados (RMSEA $\leq 0.05, \mathrm{CFI} \geq 0.95$, NNFI $\geq 0.95$; Kline, 2011). En conjunto, los resultados indican que los reactivos medidos se agrupan en los factores a los que teóricamente pertenecen y, además, que se diferencian suficientemente reflejando una estructura de covarianza congruente con las escalas utilizadas.

Con respecto a las pruebas de invarianza, se encontró que los constructos fueron esencialmente equivalentes en términos configurales y métricos entre las dos muestras (modelo de equivalencia métrica RMSEA $=0.05, \mathrm{CFI}=0.93, \Delta \mathrm{CFI}$ [solución configural - métrica] $=0.03$ ). Estos resultados se muestran en la Tabla 1.

\subsection{Descriptivos}

La Tabla 2 muestra la media (M), la desviación estándar (DE) y las correlaciones bivariadas (r) entre las variables. Como se observa, la media de estilo explicativo pesimista es más alta en Estados Unidos que en México, aunque la diferencia no es significativa $(t=-0.55, \mathrm{p}>0.05)$. También se observa que la media de autoeficacia ocupacional es más alta en México que en Estados Unidos y que la diferencia es significativa $(t=4.44, \mathrm{p}<0.01)$. La correlación entre estilo explicativo pesimista y autoeficacia ocupacional es más negativa en EE.UU. que en México. 
- Revista de Ciencias Sociales y Humanidades. ISSN-P: 0188-9834 ISSN-E:2395-8669.

Tabla 1. Pruebas de invarianza

\begin{tabular}{lcccccc}
\hline Modelo / Indicador & $\chi^{2}$ & df & RMSEA & CFI & $\Delta \chi^{2}$ & $\Delta$ CFI \\
\hline Equivalencia configural & 1025,73 & 352 & 0,050 & 0,900 & & \\
Equivalencia métrica & 967,67 & 351 & 0,050 & 0,930 & $58,06 * *$ & 0,03 \\
\hline$* *=p<0.010$ & & & & & &
\end{tabular}

Fuente: Elaboración propia

Tabla 2. Descriptivos y correlaciones bivariadas

\begin{tabular}{|c|c|c|c|c|c|c|c|c|c|}
\hline \multirow[b]{2}{*}{ Variable } & \multicolumn{2}{|c|}{$M$} & \multicolumn{2}{|c|}{$D E$} & \multirow{2}{*}{$t$} & \multicolumn{4}{|c|}{$r$} \\
\hline & México & EE.UU. & México & EE.UU. & & México & & EE.UU. & \\
\hline Estilo explicativo pesimista & 3.72 & 3.76 & 0.90 & 0.78 & -0.55 & 016 & $* *$ & 020 & $* *$ \\
\hline Autoeficacia ocupacional & 5.95 & 5.61 & 0.80 & 1.00 & $4.44^{* *}$ & & & & \\
\hline
\end{tabular}

Fuente: Elaboración propia

\subsection{V arianza del método común}

Una vez controlando por los efectos del método, las correlaciones entre el estilo explicativo pesimista y la autoeficacia ocupacional variaron muy poco $(\Delta \mathrm{r}$ México $=0.06, \Delta$ r EE.UU. $=0.04)$. Sin embargo, el nivel de significatividad de las correlaciones permaneció igual indicando que la varianza del método común no es una amenaza grave a la validez de los resultados obtenidos.

\subsection{Prueba de hipótesis}

La Hipótesis 1 indica que el estilo explicativo pesimista se relaciona negativamente con la autoeficacia ocupacional. En la Tabla 3, en el modelo de efecto principal en la muestra completa, se observa que después de controlar por la edad, el sexo y la situación laboral de los respondientes, el estilo explicativo pesimista tiene una relación negativa y significativa con la autoeficacia ocupacional. Adicionalmente, el modelo de efecto principal explica un 3.57 \% más de varianza significativa que el modelo en la muestra completa que incluye sólo a los controles. Con base en esta evidencia se acepta la Hipótesis 1. 
Tabla 3. Coeficientes de regresión para la autoeficacia ocupacional

\begin{tabular}{|c|c|c|c|c|c|}
\hline Variables & $\begin{array}{c}\text { Muestra completa } \\
\text { (Controles) }\end{array}$ & $\begin{array}{l}\text { Muestra completa } \\
\text { (Efecto principal) }\end{array}$ & $\begin{array}{l}\text { Muestra completa } \\
\text { (Moderación) }\end{array}$ & México & EE.UU. \\
\hline \multirow[t]{2}{*}{ Constante } & $5.575^{* *}$ & $6.339 * *$ & $6.44 * *$ & $6.581^{* *}$ & $6.411^{* *}$ \\
\hline & $(0.226)$ & $(0.277)$ & $(0.310)$ & $(0.362)$ & $(0.426)$ \\
\hline \multirow[t]{2}{*}{ Edad } & $0.014^{*}$ & $0.016^{* *}$ & $0.012 *$ & 0.010 & 0.012 \\
\hline & $(0.005)$ & $(0.005)$ & $(0.005)$ & $(0.008)$ & $(0.008)$ \\
\hline \multirow[t]{2}{*}{ Sexo } & -0.121 & $-0.151^{*}$ & $-0.176^{*}$ & $-0.272 * *$ & -0.104 \\
\hline & $(0.076)$ & $(0.076)$ & $(0.075)$ & $(0.095)$ & $(0.116)$ \\
\hline \multirow[t]{2}{*}{ Situación laboral } & $-0.514^{* *}$ & $-0.513^{* *}$ & $-0.420^{* *}$ & 0.195 & $-0.605^{* *}$ \\
\hline & $(0.132)$ & $(0.130)$ & $(0.131)$ & $(0.242)$ & $(0.165)$ \\
\hline \multirow[t]{2}{*}{ Estilo explicativo pesimista } & & $-0.205^{* *}$ & $-0.161^{* *}$ & $-0.147^{* *}$ & $-0.245^{* *}$ \\
\hline & & $(0.044)$ & $(0.058)$ & $(0.052)$ & $(0.075)$ \\
\hline \multirow[t]{2}{*}{ Dummy por país } & & & $-0.277^{* *}$ & & \\
\hline & & & $(0.076)$ & & \\
\hline \multirow[t]{2}{*}{ Estilo explicativo pesimista $x$ dummy por país } & & & -0.091 & & \\
\hline & & & $(0.090)$ & & \\
\hline$F$ & $8.92 * *$ & $12.21 * *$ & $10.63 * *$ & $4.62 * *$ & $7.32 * *$ \\
\hline$R^{2}$ & $4.61 \%$ & $8.18 \%$ & $10.46 \%$ & $6.32 \%$ & $9.81 \%$ \\
\hline$R^{2}$ ajustada & $4.09 \%$ & $7.51 \%$ & $9.48 \%$ & $4.95 \%$ & $8.47 \%$ \\
\hline$\Delta R^{2}$ & $4.09 \%$ ** & $3.57 \%$ ** & $2.28 \% * *$ & $2.96 \% \%^{* *}$ & $3.59^{* *}$ \\
\hline
\end{tabular}

La Hipótesis 2 sugiere que el estilo explicativo pesimista está relacionado más negativamente con la autoeficacia ocupacional en Estados Unidos que en México. En la Tabla 3, en el modelo de moderación en la muestra completa, se observa que la interacción entre el estilo explicativo pesimista y la dummy por país no es significativa una vez controlando por la dummy. Y aunque este modelo explica un $2.28 \%$ más de varianza significativa que el modelo de efecto principal, en primera instancia, la Hipótesis 2 debería ser rechazada. Sin embargo, dado que la dummy por país es significativa en el modelo de moderación, el valor $\mathrm{p}$ de la interacción no es confiable pues de entrada ( $\mathrm{y}$ sin tomar en cuenta el efecto interactivo del estilo explicativo pesimista), ya existen diferencias significativas en la autoeficacia ocupacional por país (También véase el valor $\mathrm{p}$ del estadístico $t$ de la autoeficacia ocupacional en la Tabla 2).

Por lo anterior, se realizó la prueba de Chow para verificar si los coeficientes de regresión del estilo explicativo pesimista son esencialmente iguales entre los países analizados. El valor de la prueba de Chow $(F=6.95, \mathrm{p}<0.010)$ indica que existen diferencias significativas en cuanto a la relación del estilo explicativo pesimista con la autoeficacia ocupacional entre México y Estados Unidos. Para más detalle, en la Tabla 3, se puede observar en los modelos individuales por país que el coeficiente del estilo explicativo pesimista es sensiblemente más negativo en Estados Unidos $(\beta=-0.245, \mathrm{p}<0.010)$ que en México $(\beta=$ $-0.147, \mathrm{p}<0.010)$. Además, el modelo que incluye al estilo explicativo pesimista en la muestra de EE.UU. explica más varianza adicional significativa respecto a un modelo base que sólo incluye a los controles $\left(\Delta R^{2}=3.59 \%\right)$ que su contraparte en la muestra de México $\left(\Delta R^{2}=2.96 \%\right)$. En conjunto, esta evidencia brinda soporte suficiente para la Hipótesis 2. 
Ante la posibilidad de que el coeficiente significativo de diferentes variables (p.ej. sexo y estatus laboral) en las dos muestras analizadas pudiera alterar los resultados de la regresión y, con ello, el valor de la prueba de Chow, en un análisis adicional se eliminaron los controles y se regresó de forma independiente a la autoeficacia sobre el estilo explicativo en cada una de las muestras. Con estos resultados se volvió a correr la prueba de Chow y el resultado continuó siendo significativo $(\mathrm{p}<0.010)$, indicando diferencias entre las muestras con respecto al impacto del estilo explicativo pesimista en la autoeficacia ocupacional. Dado que en este análisis se elimina la posibilidad de interacción entre las variables de la regresión múltiple, los resultados brindan más confianza sobre la prueba de la Hipótesis 2 y su interpretación. Sin embargo, como se indica más adelante, estudios posteriores deberían confirmar los resultados utilizando muestras con mayor grado de equivalencia en cuanto a variables como el sexo y la situación laboral.

\section{Discusión}

Los resultados de este estudio indican que el estilo explicativo pesimista tiene una relación negativa con la autoeficacia ocupacional y que esta relación es más fuerte en Estados Unidos que en México. Estos hallazgos apoyan y extienden el conocimiento actual sobre las consecuencias del estilo explicativo pesimista y los factores contextuales (p.ej. cultura) que pueden pronunciar su efecto en variables psicológicas de impacto en las organizaciones.

Investigaciones anteriores sugieren que el estilo atribucional (explicativo) afecta el esfuerzo de los empleados y la satisfacción laboral (Corr y Gray, 1996). Incluso se ha vislumbrado que el estilo atribucional puede influenciar la rotación de personal (Proudfoot et al., 2009). Sin embargo, el estudio de la manera en que el estilo explicativo influye sobre variables organizacionales aún debe profundizarse. En particular, se debe investigar más sobre el impacto del estilo explicativo pesimista en variables antecedentes de la satisfacción laboral, el esfuerzo del personal y las intenciones de renuncia. Por ello, resulta importante indagar sobre la relación entre el estilo explicativo pesimista y la autoeficacia ocupacional. Está documentado que la autoeficacia es un antecedente crítico tanto de la satisfacción y del desempeño laboral (Judge y Bono, 2001), como de las intenciones de renuncia (Park y Jung, 2015). Esta investigación ayuda a cubrir esta brecha en el conocimiento pues indica que el estilo explicativo pesimista se relaciona negativamente con la autoeficacia ocupacional. Esto es, entre más internas, estables y globales sean las atribuciones de los eventos negativos (p.ej. fracasos) que realizan las personas, menos propensas serían a las experiencias directas de éxito que les hagan desarrollar creencias de autoeficacia en y para el desempeño exitoso de una ocupación.

Los hallazgos de este trabajo también respaldan y extienden el conocimiento actual sobre el efecto de la cultura nacional en la relación entre constructos psicológicos y laborales. McCrae (2000) indica que la cultura interactúa con los rasgos personales para afectar el comportamiento. De tal suerte, las diferencias culturales entre México y varios países afectan las relaciones entre constructos psicológicos (p.ej. Cernas-Ortiz y Davis, 2016). Los resultados del presente trabajo respaldan esta línea de evidencia pues también indican que la cultura nacional modera la relación entre rasgos personales (p.ej. estilo explicativo) y variables organizacionales (p.ej. autoeficiacia ocupacional). No obstante, y expandiendo el conocimiento más allá de investigaciones anteriores, los hallazgos de este estudio sugieren que una cul- 
tura baja en orientación al corto plazo e indulgencia (p.ej. EE.UU.) puede amplificar el efecto pernicioso de las atribuciones malsanas de eventos negativos en la posibilidad de tener experiencias directas de éxito que conduzcan a una autoeficacia ocupacional sólida.

Sin embargo, se debe tener en cuenta que los resultados de este trabajo no implican que en la cultura mexicana una menor propensión a hacer atribuciones negativas de eventos negativos aumente necesariamente los niveles de autoeficacia ocupacional. Oettingen (1995b) argumenta que dado que en las culturas colectivistas (p.ej. México) la autoeficacia está más influenciada por lo que un grupo social relevante piense del desempeño del individuo que lo que él/ella mismo(a) piense al respecto, en las culturas individualistas (p.ej. EE.UU.) las experiencias directas de éxito se traducen más en autoeficacia que en las colectivistas. Por lo tanto, aunque en un ambiente individualista las atribuciones negativas con respecto al fracaso podrían hundir más severamente la autoeficacia, en un ambiente sociocéntrico no necesariamente una menor tendencia a hacer atribuciones negativas de eventos negativos (o incluso atribuciones positivas de éxito) se traduciría en una mayor autoeficacia ocupacional.

\subsection{Limitaciones}

Una limitación de esta investigación es que los participantes contaban con un nivel educativo alto (maestría) y que no se recolectó información más detallada sobre su experiencia laboral (p.ej. años en la organización o en el puesto). Por ello, los resultados se circunscriben con mayor precisión a empleados administrativos o técnicos especializados con una alta preparación académica. También es importante resaltar que los datos fueron obtenidos de regiones particulares de México y Estados Unidos. Por lo tanto, aunque cierta evidencia indica que la cultura de cualquiera de estos países es suficientemente homogénea como para diferenciarse de la de sus vecinos (Minkov y Hofstede, 2012), la generalización a la población entera de trabajadores de ambos países debe ser cautelosa. También es una limitación el comparar las muestras de dos países suponiendo que las diferencias culturales existen sin en realidad medir los valores que podrían diferir entre ellos.

\section{2. Áreas futuras de investigación}

Para comprender mejor el tema tratado, investigaciones futuras deberían indagar más sobre si la cultura mexicana (en comparación con la estadounidense o la de otros países), fomenta un menor rango en las puntuaciones de autoeficacia ocupacional. Como se implica de los resultados de esta investigación, si bien la cultura de los mexicanos amortiguaría un poco las atribuciones perniciosas de eventos negativos, también, y dado su carácter eminentemente colectivista, podría inhibir la conversión de las experiencias directas de éxito en sentimientos sólidos de autoeficacia. De hecho, en la Tabla 2, la desviación estándar de la autoeficacia ocupacional es más pequeña en México que en EE.UU. Dado que, en comparación con los ciudadanos de culturas colectivistas (p.ej. Japón), los estadounidenses son más proclives al sesgo de 
autoservicio (tomar más crédito personal por los éxitos que por los fracasos) (Heine y Hamamura, 2007), ellos desarrollarían sentimientos más sólidos de autoeficacia que los mexicanos cuando sus niveles de atribuciones negativas son bajos. Esto es algo que investigaciones futuras deberían aclarar. También es importante que investigaciones futuras midan los valores culturales de los encuestados e incluyan estas mediciones en los análisis en lugar de suponer diferencias a priori entre los países. Por supuesto que investigaciones posteriores deberían analizar muestras más grandes, con mayor equivalencia en características demográficas y, preferentemente, que involucren varias regiones de los países analizados.

\subsection{Implicaciones prácticas}

La autoeficacia ocupacional es importante para personas y organizaciones pues es un recurso psicológico que ayuda a afrontar el estrés y, por lo tanto, a fomentar la salud ocupacional y mejorar la productividad (Luthans, Youseff-Morgan y Avolio, 2015). Por lo tanto, en la medida en que las organizaciones promuevan el debilitamiento del estilo explicativo pesimista, también podrían fomentar que los empleados mejoren su nivel de bienestar y, con ello, la efectividad organizacional. Una ruta para esto es el entrenamiento cognitivoconductual (Seligman, Castellon, Cacciola et al., 1988). Aunque el estilo explicativo se considera un atributo individual relativamente estable, Proudfoot et al. (2009) encontraron que un entrenamiento cognitivo-conductual puede modificar el estilo atribucional en el ambiente laboral. Notablemente, en aras de nivelar los niveles de autoeficacia ocupacional entre su personal, los gerentes de empresas con operaciones en los países considerados en este estudio podrían dar más importancia al entrenamiento en cuestión en Estados Unidos.

\section{Conclusión}

El estilo explicativo pesimista puede afectar a la autoeficacia ocupacional. Este fenómeno puede amplificarse por los valores culturales de algunas sociedades. En la medida en que el estilo atribucional en mención pueda ser modificado, también se podría fomentar una mayor autoeficacia ocupacional en los empleados de las organizaciones mexicanas y, con mayor énfasis, las estadounidenses. La mejora en la autoeficacia ocupacional tiene el potencial de elevar el bienestar individual y la efectividad de las organizaciones.

\section{Referencias}

Abramson, Lyn, Martin Seligman y John Teasdale. 1978. Learned helplessness in humans: Critique and reformulation. Journal of Abnormal Psychology, 87 (1): 49-74. https://doi.org/10.1037//0021-843x.87.1.49 Bandura, Albert. 1977. Self-efficacy: Toward a unifying theory of behavioral change. Psychological Review, 34 (2): 191-252. https://doi.org/10.1037//0033-295x.84.2.191

Bandura, Albert y Edwin Locke. 2003. Negative self-efficacy and goal effects revisited. Journal of Applied Psychology, 88 (1): 87-99. https://doi.org/10.1037/0021-9010.88.1.87

Bridges, Robert. 2001. Using attributional style to predict academic performance: How does it compare to traditional methods? Personality and Individual Differences, 31: 723-730. https://doi.org/10.1016/ s0191-8869(00)00174-4 
Byrne, Barbara. 2006. Structural equation modeling with LISREL, PRELIS, and SIMPLIS: Basic concepts, applications, and programming. Nueva Jersey: Lawrence Erlbaum Associates, Publishers.

Cernas-Ortiz, Daniel y Mark Davis. 2016. Future and past negative time perspective influences on job satisfaction and organizational commitment in Mexico and the United States. Management Research: Journal of the Iberoamerican Academy of Management, 14 (3): 317-338. https://doi.org/10.1108/mrjiam-04-2016-0665

Cheng, Helen y Adrian Furnham. 2001. Attributional style and personality as predictor of happiness and mental health. Journal of Happiness Studies, 2: 307-327.

Corr, Philip y Jeffrey Gray. 1996. Attributional style as a personality factor in insurance sales performance in the UK. Journal of Occupational and Organizational Psychology, 69: 83-87. https://doi. org/10.1111/j.2044-8325.1996.tb00601.x

Cortés, Carlos. 1989. Cómo ver al vecino: El libro de texto hollywoodense sobre México. En Imágenes de México en Estados Unidos, coordinado por J. Coatsworth y C. Rico. México: Fondo de Cultura Económica, 115-144.

Curzio, Leonardo. 2013. Las relaciones México-Estados Unidos, 1756-2010. Norteamérica, 8 (2): 201 214. https://doi.org/10.1016/s1870-3550(13)71779-8

Diener, Ed y Marissa Diener. 1995. Cross-cultural correlates of life satisfaction and self-esteem. Journal of Personality and Social Psychology, 68 (4): 653-663. https://doi.org/10.1007/978-90-481-2352-0_4

Furnham, Adrian, Valda Sadka y Chris Brewin. 1992. The development of an occupational attributional style questionnaire. Journal ofOrganizational Behavior, 13:27-39. https://doi.org/10.1002/job.4030130104 Gits, Marilyn y Terrence Mitchell. 1992. Self-efficacy: A theoretical analysis of its determinants and malleability. Academy of Management Journal, 17 (2): 183-211. https://doi.org/10.2307/258770

Heine, Steven y Takeshi Hamamura. 2007. In search of East Asian self-enhancement. Personality and Social Psychology Review, 11: 1-24. https://doi.org/10.1177/1088868306294587

Hofstede, Gert. 2015. Culture's causes: The next challenge. Cross-Cultural Management, 22 (4): 545-569. https://doi.org/10.1108/ccm-03-2015-0040

Hofstede, Geert, Gert Hofstede y Michael Minkov. 2010. Cultures and organizations. Software of the Mind. New York: McGrawHill

House, Robert, Paul Hanges, Mansour Javidan, Peter Dorfman y Vipin Gupta. 2004. Culture, leadership and societies. The GLOBE study of 62 societies. Thousand Oaks: SAGE Publications.

Judge, Timothy y Joyce Bono. 2001. Relationship of core self-evaluations traits -self-esteem, generalized self-efficacy, locus of control, and emotional stability- with job satisfaction and job performance: A metaanalysis. Journal of Applied Psychology, 86 (1): 80-92. https://doi.org/10.1037//0021-9010.86.1.80

Kline, Rex. 2011. Principles and practice of structural equation modeling. Nueva York: Guilford Press.

Lindell, Michael y David Whitney. 2001. Accounting for common method variance in cross-sectional research designs. Journal of Applied Psychology, 86 (1): 114-121. http://dx.doi.org/10.1037/00219010.86.1.114

Locke, Edwin y Gary Latham. 2009. Has goal setting gone wild, or have its attackers abandoned good scholarship? Academy of Management Perspectives, 23 (1): 17-23. https://doi.org/10.5465/amp.2009.37008000 Luthans, Fred, Carolyn Youssef-Morgan y Bruce Avolio. 2015. Psychological capital and beyond. Nueva York: Oxford University Press 
McCrae, Robert. 2000. Trait psychology and the revival of personality and culture studies. American Behavioral Scientist, 44: 10-31. https://doi.org/10.1177/00027640021956062

Merino-Tejedor, Enrique, Manuel Fernández-Ríos y Mariana Bargsted-Aravena. 2015. El papel moderador de la autoeficacia ocupacional entre la satisfacción y la irritación laboral. Universitas Psychologica, 14 (1): 15-25. https://doi.org/10.11144/javeriana.upsy14-1.pmao

Minkov, Michael. 2009. Predictors of differences in subjective well-being across 97 nations. Cross-Cultural Research, 43 (2): 152-179. https://doi.org/10.1177/1069397109332239

Minkov, Michael y Geert Hofstede. 2012. Is national culture a meaningful concept? Cultural values delineate homogeneous national clusters of in-country regions. Cross-Cultural Research, 46 (2): 133-159. https://doi.org/10.1177/1069397111427262

Miralles, Cibeles y José Navarro. 2016. Relaciones asimétricas entre atribuciones y afecto en el trabajo. Revista de Psicología del Trabajo y de las Organizaciones, 32 (1): 55-65. https://doi.org/10.1016/j. rpto.2015.11.003

OECD Better Life Index. 2017. Satisfacción ante la vida. http://www.oecdbetterlifeindex.org/es/topics/ life-satisfaction-es/ (17 de julio, 2017).

Oettingen, Gabriele. 1995a. Explanatory style in the context of culture. En Explanatory Style, editado por G. Buchanan y M. Seligman. New Jersey: Erlbaum, 209-224.

Oettingen, Gabriele. 1995b. Cross-cultural perspectives on self-efficacy. En Self-efficacy in Changing Societies, editado por A. Bandura. New York: Cambridge University Press, 149-176.

Park, In-Jo y Heajung Jung. 2015. Relationships among future time perspective, career and organizational commitment, occupational self-efficacy and turnover intention. Social Behavior and Personality, 43 (9): 1547-1562. https://doi.org/10.2224/sbp.2015.43.9.1547

Paz, Octavio. 2009. El Laberinto de la soledad. Postdata, vuelta al laberinto de la soledad. México: Fondo de Cultura Económica.

Peterson, Christopher y Martin Seligman. 1984. Causal explanations as a risk factor for depression: Theory and evidence. Psychological Review, 91: 347-374. https://doi.org/10.1037/0033-295x.91.3.347 Proudfoot, Judith, Philip Corr, David Guest y Graham Dunn. 2009. Cognitive-behavioral training to change attributional style improves employee well-being, job satisfaction, productivity, and turnover. Personality and Individual Differences, 46: 147-153. https://doi.org/10.1016/j.paid.2008.09.018

Rigotti, Thomas, Birgit Schyns y Gisela Mohr. 2008. A short version of the occupational self-efficacy scale: Structural and construct validity across five countries. Journal of Career Assessment, 16 (2): 238255. https://doi.org/10.1177/1069072707305763

Schyns, Birgit y Gernot von Collani. 2002. A new occupational self-efficacy scale and its relation to personality constructs and organizational variables. European Journal of Work and Organizational Psychology, 2: 219-241. https://doi.org/10.1080/13594320244000148

Seligman, Martin. 2006. Learned optimism. How to change your mind and your life. Nueva York: Vintage Books Seligman, Martin, Camilo Castellon, John Cacciola, Peter Schulman, Lester Luborsky, Maxine Ollove y Robert Downing. 1988. Explanatory style change during cognitive therapy for unipolar depression. Journal of Abnormal Psychology, 97: 13-18. https://doi.org/10.1037//0021-843x.97.1.13

Spector, Paul. 1982. Behavior in organizations as a function of employees' locus of control. Psychological Bulletin, 91, 482-497. https://doi.org/10.1037/0033-2909.91.3.482 
- Revista de Ciencias Sociales y Humanidades. ISSN-P: 0188-9834 ISSN-E:2395-8669.

Urien, Begoña, Amparo Osca y Lourdes García-Salmones. 2017. Role ambiguity, group cohesion and job satisfaction: A demands-resources model (JD-R) study form Mexico and Spain. Revista Latinoamericana de Psicología, 49: 137-145. https://doi.org/10.1016/j.rlp.2015.09.014

Weiner, Bernard. 2010. The development of an attribution-based theory of motivation: A history of ideas. Educational Psychologist, 45 (1): 28-36. https://doi.org/10.1080/00461520903433596 